\title{
A role for abdominal ultrasound in discriminating suspected necrotizing enterocolitis in congenital heart disease patients
}

\author{
Stefanie P. Lazow ${ }^{1}$ (D) Sarah A. Tracy ${ }^{1} \cdot$ Judy A. Estroff ${ }^{2} \cdot$ Richard B. Parad $^{3} \cdot$ Ilse M. Castro-Aragon $^{4} \cdot$ Alan M. Fuji $^{5}$. \\ Steven J. Staffa ${ }^{1}$. David Zurakowski ${ }^{1}$. Catherine Chen ${ }^{1}$
}

Accepted: 20 September 2021 / Published online: 28 September 2021

(c) The Author(s), under exclusive licence to Springer-Verlag GmbH Germany, part of Springer Nature 2021

\begin{abstract}
Purpose We evaluated the diagnostic utility of abdominal ultrasound (AUS), an adjunct to abdominal X-ray (AXR), for necrotizing enterocolitis (NEC) in congenital heart disease (CHD) patients.

Methods 86 patients with suspected NEC from 2009 to 2018 were classified as with CHD $(n=18)$ if they required cardiac intervention versus without CHD $(n=68)$. Clinical and radiological data were collected, including AXR and AUS concordance. Wilcoxon rank-sum test and Fisher's exact test were performed.

Results CHD patients had higher birth weights $(p<0.001)$ and gestational ages $(p<0.001)$ than non-CHD patients. CHD patients presented more frequently with hypotension $(p=0.041)$ and less frequently with bilious emesis $(p<0.001)$. Overall, CHD patients were less likely to have AUS findings of pneumatosis (33.3 vs. $72.1 \% ; p=0.005)$ and decreased mural flow $(0$ vs. $20.6 \% ; p=0.035$ ) compared to non-CHD patients. On concordance analysis, CHD patients had 3.9-fold more discordant studies with pneumatosis on AXR but not on AUS (33.3 vs. 8.8\%; $p=0.016)$ compared to non-CHD patients. Urgent surgery was required in $5.6 \%$ of CHD patients versus $16.2 \%$ of non-CHD patients.

Conclusion CHD patients with suspected NEC represent a distinct clinical population. AUS has particular utility in assessing findings of bowel viability in the CHD NEC population, reflecting reduced rates of surgical NEC.
\end{abstract}

Keywords Necrotizing enterocolitis $\cdot$ Abdominal ultrasound $\cdot$ Abdominal radiograph $\cdot$ Cardiac $\cdot$ Congenital heart disease $\cdot$ Pneumatosis

This work was presented at the Massachusetts Chapter of the American College of Surgeons Annual Meeting (Virtual), December, 2020.

Stefanie P. Lazow

slazow@bidmc.harvard.edu

$\triangle$ Catherine Chen

catherine.chen@childrens.harvard.edu

1 Department of Surgery, Boston Children's Hospital/Harvard Medical School, 300 Longwood Avenue - Fegan 3, Boston, MA 02115, USA

2 Department of Radiology, Boston Children's Hospital/Harvard Medical School, Boston, MA, USA

3 Department of Pediatric Newborn Medicine, Brigham and Women's Hospital/Harvard Medical School, Boston, MA, USA

4 Department of Radiology, Boston Medical Center/Boston University School of Medicine, Boston, MA, USA

5 Department of Pediatrics, Boston Medical Center/Boston University School of Medicine, Boston, MA, USA

\author{
Abbreviations \\ AUS Abdominal ultrasound \\ ASD Atrial septal defect \\ AXR Abdominal radiograph \\ CHD Congenital heart disease \\ IQR Interquartile range \\ LOS Length of stay \\ NEC Necrotizing enterocolitis \\ NICU Newborn intensive care unit \\ PDA Patent ductus arteriosus \\ PPD Primary peritoneal drainage
}

\section{Introduction}

Necrotizing enterocolitis (NEC), characterized by inflammation and necrosis of the bowel, is most commonly a disease of the preterm neonate. However, NEC is also seen less frequently in full-term neonates, often in the setting of complex congenital heart disease (CHD) [1-3]. The development of 
NEC in CHD patients may be related to bowel hypoperfusion [4] and is most frequently reported in CHD patients with single ventricle physiology [5-7]. In addition to the previously reported different demographics of CHD and non-CHD patients with NEC, reduced rates of surgical NEC have also been reported in CHD patients. This finding reflects decreased rates of full-thickness bowel ischemia and bowel perforation in the CHD population [1, 8]. CHD patients with surgical NEC have also been shown to present with more frequent colonic involvement compared to nonCHD patients, potentially highlighting a different underlying pathophysiology [9].

Despite the different clinical course of CHD patients with suspected NEC, there have been few studies focusing on the role of specific radiological findings in evaluating for suspected NEC in CHD patients. Abdominal ultrasound (AUS) has emerged as a valuable diagnostic adjunct to abdominal X-ray (AXR) in the general NEC population [10]. Despite the limitations associated with its user-dependent nature, AUS plays an important role in the setting of indeterminate or non-specific AXR findings during an evaluation for NEC $[10,11]$ with the potential benefit of reducing radiation exposure from serial AXR. AUS, like AXR, can assess for pneumatosis, portal venous gas, and pneumoperitoneum. It can also uniquely provide a detailed evaluation of the character and volume of free intra-abdominal fluid, identify focal fluid collections, and assess bowel wall thickness, peristalsis, and perfusion [12-17]. Recent studies have suggested that AUS may have increased sensitivity for bowel ischemia compared with AXR [18, 19]. More detailed bowel assessment may be particularly helpful in ruling out bowel ischemia in CHD patients with a lower clinical suspicion for surgical disease.

In this study, we primarily aimed to evaluate if AUS plays a unique role in discriminating suspected NEC specifically in the CHD population. First, we compared demographic and clinical features of patients with and without CHD undergoing evaluation for suspected NEC. Next, we compared radiological findings over the admission as well as concordance between paired AUS and AXR for findings of pneumatosis, portal venous gas, and pneumoperitoneum in patients with and without CHD. Lastly, clinical outcomes including rates of surgical NEC were compared between patients with and without CHD.

\section{Materials and methods}

This is an IRB-approved multicenter retrospective review performed at three level III-IV newborn intensive care units (NICUs) in Boston, Massachusetts with pediatric surgery consultation at all three sites provided by Boston Children's Hospital. Data were collected and managed using REDCap
(Research Electronic Data Capture), which is a secure, webbased software platform designed to support research data capture studies [20, 21].

Medical records of patients undergoing imaging evaluation and surgical consultation with concern for NEC from 2009 to 2018 were reviewed for demographic, clinical, and imaging data. To be included in the study, patients had at least one paired AXR and AUS occurring within a 24-h period to facilitate concordance analysis. AUS was performed at the discretion of the provider and was not performed routinely in all patients undergoing evaluation for NEC. Demographic, clinical, and imaging data were compared between patients with and without CHD. Patients were classified as having CHD if they had structural or functional cardiac disease (including severe cardiomyopathy with heart failure) that required open surgical or catheter-based intervention, excluding isolated patent ductus arteriosus (PDA). Details of specific structural anomalies were collected. In the non-CHD cohort, the presence of structural anomalies not requiring intervention was recorded. A sub-analysis was performed comparing non-CHD patients who did or did not require PDA ligation.

Additional demographic and clinical characteristics were compared between CHD and non-CHD patients. Outborn status was defined as a patient requiring transfer from an outside hospital to one of the three NICUs. Risk factors for NEC, signs or symptoms of NEC prompting imaging evaluation, and need for respiratory support at time of NEC evaluation were also compared.

Imaging findings were collected from AXR and AUS reports if the study indication included clinical concern for NEC or surveillance after previous abnormal studies with ongoing concern for NEC. Single- or two-view AXR was performed by radiology technologists and read by experienced pediatric radiologists. The two-view films were variable including supine with left lateral decubitus, supine with cross-table lateral, and prone with left lateral decubitus. AUS studies were performed by sonographers and read by pediatric radiologists at two of the three centers (Boston Children's Hospital and Boston Medical Center) or adult obstetrical radiologists at the third center (Brigham and Women's Hospital).

AXR and AUS findings were collected from studies occurring at any time point during the admission when the patient was being evaluated for NEC. These findings were compared between patients with and without CHD. Findings on either AXR and/or AUS included pneumatosis, portal venous gas, and pneumoperitoneum. Additional AUS findings included echogenic free fluid, focal fluid collection, bowel wall thickening $(\geq 2.6 \mathrm{~mm})$ or thinning $(\leq 1.1 \mathrm{~mm})$ [12], decreased bowel wall mural flow, echogenic bowel, and hyperemic bowel. Alexander et al. provide examples of these AUS imaging findings [22]. Evaluation of bowel 
peristalsis was not routinely performed at all three centers, so these findings were not included. Imaging findings were considered positive if reported at least once during the hospital course.

Concordance for the presence of pneumatosis, portal venous gas, and pneumoperitoneum between paired AXR and AUS studies was also compared between CHD and non-CHD patients. Concordance analysis was performed only on paired AXR and AUS studies occurring within a 24-h window. If multiple AXRs were performed within that 24-h period, the last AXR performed before the AUS was selected. If a patient had multiple paired studies throughout the hospital course, only the first study pair with a positive result on either AXR and/or AUS for pneumatosis, portal venous gas, and/or pneumoperitoneum was included. If the patient had no positive paired studies over the hospital course, then the first negative paired study was included. Suspicious AXR findings for pneumatosis such as "bubbly lucencies concerning for NEC" were considered positive.

Several clinical outcomes during the admission were compared between the CHD and non-CHD patients including the need for surgical intervention for NEC, NEC-related mortality, and overall mortality. Surgical intervention was classified as either acute intervention (referring to primary peritoneal drainage (PPD) alone, laparotomy alone, or PPD plus laparotomy) versus delayed surgical intervention for NEC-related stricture. The duration of the first antibiotic course for NEC treatment and time to resume full enteral feeding after initial concern for NEC were also compared.

\section{Statistical analysis}

The non-parametric Wilcoxon rank-sum test and Fisher's exact test were used, as appropriate, to compare CHD and non-CHD patient characteristics, radiological findings, and clinical outcomes on univariate analysis. Fisher's exact test was used to compare concordance categories for AXR and AUS findings between CHD and non-CHD patients. The same analyses were used to compare non-CHD patients who did versus did not undergo PDA ligation. Statistical analyses were performed using Stata version 16.0 (StataCorp, College Station, Texas). A two-sided p-value $<0.05$ was considered statistically significant.

\section{Results}

\section{Patient demographics}

In this study, 86 patients with concern for NEC had at least one paired AXR and AUS. Of the 86 patients, 68 (79.1\%) underwent single-view AXR, while 18 (20.9\%) underwent two-view AXR. For the two-view AXR, the views included supine and left lateral decubitus $(n=15$; $83.3 \%)$, supine and cross-table lateral $(n=2 ; 11.1 \%)$, and prone and left lateral decubitus $(n=1 ; 5.6 \%)$.

There were 18 patients with CHD requiring cardiac intervention. CHD anomalies requiring intervention included hypoplastic left heart syndrome $(n=5 ; 27.8 \%)$, Tetralogy of Fallot ( $n=3 ; 16.7 \%)$, severe cardiomyopathy and/or heart failure $(n=3 ; 16.7 \%)$, atrioventricular canal defect $(n=2 ; 11.1 \%)$, double outlet right ventricle $(n=2$; $11.1 \%$ ), partial anomalous pulmonary venous return with aortic coarctation $(n=1 ; 5.6 \%)$, pulmonary atresia with intact ventricular septum $(n=1 ; 5.6 \%)$, and isolated atrial septal defect (ASD) with ventricular septal defect $(n=1$; $5.6 \%)$. Of these 18 patients, 15 (83.3\%) required cardiac surgery, while $3(16.7 \%)$ required catheter-based intervention. Fifteen $(83.3 \%)$ patients required either cardiac bypass during surgery or ECMO during their hospital course. Sixteen (88.9\%) CHD patients had PDAs, and 11 (61.1\%) required PDA ligation. Of the 18 CHD patients, $14(77.8 \%)$ had their first paired AXR and AUS performed to evaluate for NEC following their cardiac intervention. This occurred at a median 53 days (IQR 8.3, 119) after cardiac intervention. In contrast, 4 (22.2\%) patients had their first paired AXR and AUS with concern for NEC performed before their cardiac intervention. This occurred at a median 23.5 days (IQR 6.3, 76.8) prior to cardiac intervention.

There were 68 patients classified as non-CHD patients. Eleven (16.2\%) patients in this cohort had patent foramen ovale or ASD, and 1 (1.5\%) patient had a bicuspid aortic valve. None of these structural defects required intervention during the hospital course. Of the non-CHD patients, 31 (45.6\%) had PDAs. Eleven (16.2\%) non-CHD patients with PDAs required medical therapy such as acetaminophen or indomethacin, and $12(17.6 \%)$ required PDA ligation.

Patient demographics and clinical characteristics are compared between the CHD and non-CHD patients in Table 1 . Compared with non-CHD patients, CHD patients had a later median gestational age at birth (37.0 vs. 27.6 weeks; $p<0.001)$ and greater median birth weight (2.4 vs. $1.0 \mathrm{~kg}$; $p<0.001)$. CHD patients also had significantly higher median 1-min APGAR scores (7.0 vs. 4.0; $p=0.021$ ) and 5 -min APGAR scores ( 8.0 vs. $7.0 ; p=0.012$ ) than non-CHD patients.

Within the non-CHD group, patients requiring PDA ligation had significantly lower median gestational age at birth (25.5 [IQR: 24.4, 26.5] vs. 28.1 [IQR: 26.1, 31.6] weeks; $p=0.001)$, birth weight $(0.7$ [IQR: $0.5,0.9$ ] vs. 1.1 [IQR: $0.7,1.5] \mathrm{kg} ; p=0.009), 1$-min APGAR scores (2 [IQR: 1 , 3.8] vs. 5 [IQR: 3,7]; $p=0.002$ ), and 5-min APGAR scores (6 [IQR: $1.3,7]$ vs. 7 [IQR: 6,8$] ; p=0.009$ ) than patients not requiring PDA ligation. 
Table 1 Demographic and clinical comparison of patients with and without congenital heart disease (CHD)

\begin{tabular}{llll}
\hline & $\begin{array}{l}\text { CHD patients } \\
(n=18)[N, \%]\end{array}$ & $\begin{array}{l}\text { Non-CHD patients } \\
(n=68)[N, \%]\end{array}$ & $p$ value $^{\mathrm{a}}$ \\
\hline Male sex & $13(72.2)$ & $37(54.4)$ & 0.193 \\
Ethnicity & $2(11.1)$ & $12(17.6)$ & $<0.001^{*}$ \\
Non- Hispanic Caucasian & $0(0)$ & $27(39.7)$ & \\
Black & $0(0)$ & $3(4.4)$ & \\
Asian & $1(5.6)$ & $5(7.4)$ & 0.050 \\
Hispanic & $1(5.6)$ & $0(0)$ & \\
Native American & $14(77.8)$ & $21(30.9)$ & \\
Other/Unknown & & $20(29.4)$ & $<0.001^{*}$ \\
Delivery method & $1(5.6)$ & $45(66.2)$ & $<0.001^{*}$ \\
Vaginal & $15(83.3)$ & $3(4.4)$ & \\
C-section & $2(11.1)$ & $1.0(0.7,1.4)$ & $0.021^{*}$ \\
N/a & $2.4(2.1,3.7)$ & $27.6(25.5,30.3)$ & $0.012^{*}$ \\
Median birth weight (kg; IQR) & $37.0(36.3,38.6)$ & & 0.430 \\
Median gestational age at birth (weeks; & & \\
IQR) & $7.0(3.5,8.0)$ & $7.0(5.0,8.0)$ & $7(10.3)$ \\
Median 1-min APGAR (IQR) & $8.0(7.5,8.5)$ & $3(16.7)$ & \\
Median 5-min APGAR (IQR) & & & \\
Outborn status & & &
\end{tabular}

$C H D$ congenital heart disease, $I Q R$ interquartile range

*Statistically significant at $p<0.05$

${ }^{\text {a }} P$ values refer to results from the Wilcoxon rank-sum test or Fisher's exact test, as appropriate

\section{NEC risk factors and clinical presentation}

Risk factors for NEC and presenting signs/symptoms at time of NEC evaluation are summarized in Table 2. Four of $18(22.2 \%)$ CHD patients received a blood transfusion within $48 \mathrm{~h}$ of initial NEC evaluation compared to 37 of $68(54.4 \%)$ non-CHD patients $(p=0.018)$. Feeding information before the time of NEC evaluation was available in all CHD patients and 66 of 68 non-CHD patients. CHD patients were significantly less likely to have ever been breast-fed than non-CHD patients $(61.1$ vs. $87.9 \%$; $p=0.015)$. CHD patients also had higher rates of any formula feeding before NEC evaluation than non-CHD patients (38.9 vs. 19.7\%), but this did not reach statistical significance $(p=0.119)$.

The most common presenting symptom in both CHD and non-CHD patients was abdominal distension (77.8 and $91.2 \%$, respectively). Compared to non-CHD patients, CHD patients were significantly more likely to present with hypotension (66.7 vs. $39.7 \%$; $p=0.041)$ and significantly less likely to present with bilious emesis (11.1 vs. $57.4 \%$; $p<0.001)$. There were no significant differences in any other presenting symptoms (Table 2). Although fewer CHD patients required invasive mechanical ventilation than nonCHD patients ( 38.9 vs. $57.4 \%$ ), there was no significant difference in the type of respiratory support required at the time of initial evaluation for NEC $(p=0.069)$.
There were no significant differences in any NEC risk factors or presenting symptoms between non-CHD patients who did versus did not require PDA ligation $(p>0.1)$.

\section{Hospital course imaging findings}

When comparing imaging findings throughout the overall hospital course (Table 3), CHD patients were significantly less likely than non-CHD patients to have pneumatosis on AUS (33.3 vs. $72.1 \% ; p=0.005)$ and decreased bowel mural flow on AUS ( 0 vs. $20.6 \%$; $p=0.035$ ).

There were no significant differences in rates of pneumatosis on AXR (66.7 vs. $79.4 \%$; $p=0.346$ ) or any other radiological findings between CHD and non-CHD patients (Table 3).

In a sub-analysis, there were no significant differences in rates of any AXR or AUS findings over the hospital course between non-CHD patients who did versus did not require PDA ligation $(p>0.3)$.

\section{Concordance analysis}

Concordance between paired AXR and AUS for pneumatosis, portal venous gas, and pneumoperitoneum was analyzed. There was no significant difference in median hours between first paired AXR and AUS for CHD patients versus 
Table 2 Comparison of necrotizing enterocolitis (NEC) risk factors and clinical presentation in patients with and without congenital heart disease (CHD)

\begin{tabular}{|c|c|c|c|}
\hline & $\begin{array}{l}\text { CHD patients } \\
(n=18)[N, \%]\end{array}$ & $\begin{array}{l}\text { Non-CHD } \\
\text { patients }(n=68) \\
{[N, \%]}\end{array}$ & $p$ value $^{\mathrm{a}}$ \\
\hline $\begin{array}{l}\text { Blood transfusion } \\
\text { within } 48 \text { h prior to } \\
\text { NEC evaluation }\end{array}$ & $4(22.2)$ & $37(54.4)$ & $0.018 *$ \\
\hline \multicolumn{4}{|c|}{ Feeding before NEC evaluation ${ }^{\mathrm{b}}$} \\
\hline $\begin{array}{l}\text { Any breast milk } \\
\text { feeding }\end{array}$ & $11(61.1)$ & $58(87.9)$ & $0.015^{*}$ \\
\hline Any formula feeding & 7(38.9) & 13(19.7) & 0.119 \\
\hline \multicolumn{4}{|c|}{ Signs/symptoms at time of initial NEC evaluation } \\
\hline $\begin{array}{l}\text { Abdominal disten- } \\
\text { sion }\end{array}$ & 14(77.8) & $62(91.2)$ & 0.207 \\
\hline $\begin{array}{l}\text { Abdominal wall } \\
\text { erythema }\end{array}$ & $2(11.1)$ & $18(26.5)$ & 0.221 \\
\hline Bilious emesis & 2(11.1) & $39(57.4)$ & $<0.001 *$ \\
\hline Hematochezia & $9(50.0)$ & $24(35.3)$ & 0.285 \\
\hline Hypotension & $12(66.7)$ & 27(39.7) & $0.041 *$ \\
\hline Oliguria & $2(11.1)$ & $18(26.5)$ & 0.221 \\
\hline \multicolumn{4}{|c|}{ Respiratory support at time of NEC evaluation } \\
\hline Ventilator & $7(38.9)$ & $39(57.4)$ & 0.069 \\
\hline $\begin{array}{l}\text { Non-invasive } \\
\text { positive pressure } \\
\text { ventilation (CPAP } \\
\text { or BIPAP) }\end{array}$ & $5(27.8)$ & $18(26.5)$ & \\
\hline $\begin{array}{l}\text { High-flow nasal } \\
\text { cannula }\end{array}$ & $1(5.6)$ & $1(1.5)$ & \\
\hline $\begin{array}{l}\text { Low-flow nasal } \\
\text { cannula }\end{array}$ & $2(11.1)$ & $0(0)$ & \\
\hline None (room air) & $3(16.7)$ & $10(14.7)$ & \\
\hline
\end{tabular}

$N E C$ necrotizing enterocolitis, $C H D$ congenital heart disease

*Statistically significant at $p<0.05$

${ }^{\text {a }} P$ values refer to results from Fisher's exact test

${ }^{\mathrm{b}} n=66$ for non-CHD patients

for non-CHD patients (3.5 h [IQR: $1.3,11.4]$ versus $5.7 \mathrm{~h}$ [IQR: $2.8,10.0])(p=0.181)$.

Figure 1 summarizes concordance data for pneumatosis between the first paired AXR and AUS in CHD and nonCHD patients. Non-CHD patients were significantly more likely to have a concordant positive paired study for pneumatosis than CHD patients (32.4 vs. $5.6 \% ; p=0.033$; Fig. 1). CHD patients had 3.9-fold more discordant paired studies with a positive AXR, but negative AUS for pneumatosis than non-CHD patients (33.3 vs. $8.8 \%$; $p=0.016$; Fig. 1 ). Rates of discordant paired studies with a negative AXR, but positive AUS for pneumatosis were similar between CHD (27.8\%) and non-CHD (35.3\%) patients ( $p=0.780$; Fig. 1 ).

For the finding of portal venous gas, there were no significant differences between the CHD and nonCHD groups in any of the AXR and AUS concordance categories $(p>0.2)$. The majority of paired studies in both the CHD patients (72.2\%) and non-CHD patients (76.5\%) were negative on both AXR and AUS for portal venous gas. There were similar rates of discordant paired studies that were negative on AXR, but positive on AUS for portal venous gas in CHD (22.2\%) and non-CHD (22.1\%) patients. One CHD patient (5.6\%) had a discordant paired study that was positive on AXR, but negative on AUS for portal venous gas. None of the non-CHD patients had this type of discordant paired imaging for portal venous gas.

For the finding of pneumoperitoneum, $100 \%$ of studies in CHD patients and $95.6 \%$ of studies in non-CHD patients were negative on both AXR and AUS. The remaining paired studies $(n=3 ; 4.4 \%)$ in non-CHD patients were discordant with AXR negative, but AUS positive for pneumoperitoneum.

\section{Clinical outcomes}

Overall, 4 of 18 (22.2\%) CHD patients and 5 of 68 (7.4\%) non-CHD patients died during the admission $(p=0.087)$. There was a $0 \%$ NEC-specific mortality rate in CHD patients versus $5.9 \%$ in non-CHD patients, though this did not reach statistical significance $(p=0.575)$. All other mortality was secondary to cardiopulmonary failure. There were no significant differences in surgical or mortality rates between non-CHD patients who did and did not require PDA ligation $(p>0.1)$.

In the CHD cohort, only 1 patient $(5.6 \%)$ required acute surgical intervention and underwent PPD. No CHD patients required acute laparotomy for NEC. In contrast, 11 of $68(16.2 \%)$ non-CHD patients required acute surgical intervention for NEC. Three (4.4\%) non-CHD patients underwent PPD alone, 6 (8.8\%) underwent laparotomy alone, and 2 (2.9\%) underwent PPD plus laparotomy. Two of $18(11.1 \%)$ CHD patients and 6 of 68 (8.8\%) non-CHD patients had delayed surgery for presumed NEC-related stricture. There was no statistically significant difference in rates of acute surgery for NEC or delayed surgery for NEC-related stricture between CHD and non-CHD patients ( $p=0.446$ and $p=0.671$, respectively).

Both CHD and non-CHD patients underwent a median of 1 (IQR: 1,2) discrete antibiotic course for NEC treatment $(p=0.426)$. There was a trend towards decreased duration of the first antibiotic course for NEC treatment in CHD patients who received a median 7 days (IQR: 5, 14) of antibiotics compared to a median 13 days (IQR: $9,14)$ in non-CHD patients $(p=0.061)$. Median time to restart full enteral feeding after initial NEC concern was 26 days (IQR: 15, 39) in CHD patients versus 31 days (IQR: $20-51)$ in non-CHD patients $(p=0.600)$. 
Table 3 Summary of hospital course abdominal X-ray (AXR) and abdominal ultrasound (AUS) findings for patients with and without congenital heart disease (CHD)

\begin{tabular}{llll}
\hline & $\begin{array}{l}\text { CHD patients }(n=18) \\
{[N, \%]}\end{array}$ & $\begin{array}{l}\text { Non-CHD patients }(n=68) \\
{[N, \%]}\end{array}$ & $p$ value $^{\mathrm{a}}$ \\
\hline Findings concerning for ischemic bowel during admission & & \\
Pneumatosis (AXR) & $12(66.7)$ & $54(79.4)$ & 0.346 \\
Pneumatosis (AUS) & $6(33.3)$ & $49(72.1)$ & $0.005^{*}$ \\
Portal venous gas (AXR) & $7(38.9)$ & $15(22.1)$ & 0.222 \\
Portal venous gas (AUS) & $4(22.2)$ & $19(27.9)$ & 0.769 \\
Bowel wall thickening (AUS) & $5(27.8)$ & $18(26.5)$ & 0.999 \\
Bowel wall thinning (AUS) & $0(0)$ & $11(16.2)$ & 0.110 \\
Echogenic bowel (AUS) & $3(16.7)$ & $11(16.2)$ & 0.999 \\
Hyperemic bowel (AUS) & $2(11.1)$ & $10(14.7)$ & 0.999 \\
Decreased mural flow (AUS) & $0(0)$ & $14(20.6)$ & $0.035^{*}$ \\
Findings concerning for bowel perforation & during admission & & \\
Pneumoperitoneum (AXR) & $2(11.1)$ & $8(11.8)$ & 0.999 \\
Pneumoperitoneum (AUS) & $0(0)$ & $7(10.3)$ & 0.337 \\
Echogenic free fluid (AUS) & $2(11.1)$ & $12(17.6)$ & 0.724 \\
Focal fluid collection (AUS) & $0(0)$ & $7(10.3)$ & 0.337 \\
\hline
\end{tabular}

$C H D$ congenital heart disease, $A X R$ abdominal X-ray, $A U S$ abdominal ultrasound

*Statistically significant at $p<0.05$

${ }^{\text {a }} P$ values refer to results from Fisher's exact test
Fig. 1 Comparison of abdominal X-ray (AXR) and abdominal ultrasound (AUS) concordance for pneumatosis in first paired studies from patients with and without congenital heart disease (CHD versus non-CHD); Asterisks denote a statistically significant difference in the proportions of studies in that concordance category between CHD and non-CHD patients $(p<0.05)$

\section{AXR and AUS Concordance for Pneumatosis}

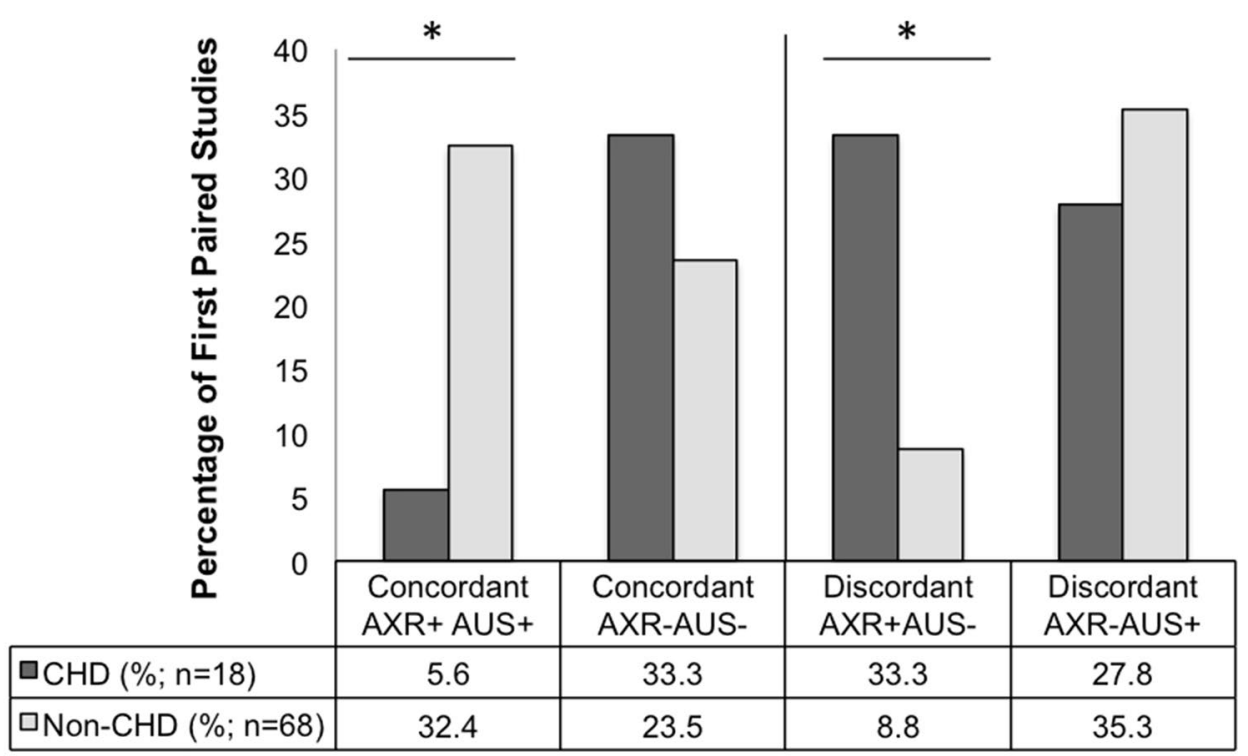

\section{Discussion}

This study's primary goal was to assess the role of AUS in discriminating suspected NEC in the unique CHD population because of ultrasound's ability to assess intraabdominal fluid collections and bowel viability [13-17].
In this study, patients with CHD undergoing evaluation for suspected NEC do appear to represent a distinct clinical population from patients without CHD. Similarly to prior studies [1, 8, 9,23], we report higher gestational ages, larger birth weights, and different risk factors for NEC development including an association with reduced breast milk feeding in the CHD compared to non-CHD 
population. Furthermore, CHD patients presented more frequently with hypotension than non-CHD patients, possibly reflecting a different underlying pathophysiology in the CHD population that may involve bowel hypoperfusion and inadequate oxygen delivery $[2,4,7]$.

This study showed the utility of AUS as an adjunct to AXR in evaluating bowel integrity in this distinct CHD patient population. Upon review of imaging findings over the entire hospital course, CHD patients were significantly less likely to have AUS findings of pneumatosis or decreased bowel mural flow compared to non-CHD patients. These results likely reflect reduced rates of full-thickness bowel ischemia in the CHD cohort. This is corroborated by the CHD population's descriptively shorter first antibiotic courses, reduced rates of acute surgical NEC, and lower NEC-related mortality compared to CHD patients, though these differences did not reach statistical significance.

Furthermore, on concordance analysis of first paired AXR and AUS studies for NEC evaluation, CHD patients had significantly fewer concordant study pairs that were both positive for pneumatosis and significantly more discordant study pairs with pneumatosis visualized on AXR, but not on AUS compared to non-CHD patients. Importantly, none of the CHD patients with pneumatosis seen on AXR, but not AUS required surgical intervention. These findings suggest that AUS may assist with ruling out full-thickness bowel ischemia in the setting of a concerning or indeterminate AXR combined with low suspicion based on a patient's clinical status. The added information from AUS may help to potentially reduce the duration of unnecessary antibiotic therapy, allow earlier initiation of feeding, and prompt earlier workup of possible alternate diagnoses such as milk protein intolerance. Both CHD and non-CHD patients had a similar proportion of paired studies with negative AXR but positive AUS for pneumatosis on concordance analysis. This finding suggests that AUS may also have increased sensitivity for pneumatosis compared to AXR in certain settings in both patient populations, as has been previously reported [18].

These findings highlight that AUS adds diagnostic value as an adjunct to AXR in the evaluation of NEC. AUS does have several limitations, however. AUS is more time intensive than AXR and, therefore, may not be appropriate for an unstable patient. Furthermore, AUS is user dependent and requires an experienced imager. The results from our centers with radiologists who have years of experience in sonography of newborns may, therefore, not be generalizable to other centers.

In this study, we did not include patients with isolated PDA in the CHD cohort even if they required PDA ligation based upon precedent set in prior literature [9] and lack of consensus for indications for treatment of PDA [24]. However, we did perform a sub-analysis of patients in the non-CHD cohort who required PDA ligation given the reported association between patients with PDAs and increased NEC risk [25-27]. There were no significant differences in any clinical or imaging findings between nonCHD patients who did versus did not require PDA ligation. Future studies should further evaluate NEC risk and the role of AUS in this particular patient cohort.

There are several overall study limitations. First, this was a retrospective study with small group sizes, especially in the CHD cohort. The CHD cohort represented a heterogeneous group of patients with different structural and functional cardiac lesions; this variation has implications for possible differences in hemodynamic derangement leading to bowel hypoperfusion. The majority of CHD patients received care at only one of the three centers (Boston Children's Hospital), which may have biased radiological comparisons given each center had a different radiology team performing and reading images. In addition, only patients with paired AXR and AUS were included to facilitate concordance analysis. Thus, patients who were followed with only serial AXR without AUS were excluded from the study. Finally, other clinical data such as laboratory values, details of antibiotic and pressor medications, and the pathological location of necrotizing enterocolitis at surgery were not routinely collected and were not included in the analysis.

Future prospective studies are necessary to determine if the addition of AUS to AXR can influence management decisions regarding antibiotic duration and time to re-initiation of feeding, stratify surgical risk, and ultimately improve clinical outcomes in both CHD and non-CHD patients with suspected NEC.

\section{Conclusions}

CHD patients with suspected NEC appear to represent a distinct clinical population compared to non-CHD patients with later gestational ages at birth, larger birth weights, and an increased likelihood of experiencing hypotension at the time of initial workup for NEC. In the distinct CHD patient population with reduced rates of surgical NEC, AUS has particular utility in demonstrating a lower incidence of pneumatosis and decreased bowel mural flow compared to nonCHD patients.

Acknowledgements The authors would like to thank Dr. Jennifer Perez MD for her assistance with data collection.

Author contributions SL: data collection, data analysis, manuscript drafting, manuscript review. ST: study design, data collection, data analysis, manuscript review. JE: study design, manuscript review. RP: study design, manuscript review. ICA: study design, manuscript review. AF: study design, manuscript review. SS: data analysis, manuscript review. DZ: study design, data analysis, manuscript review. CC: study design, data analysis, manuscript review. 
Funding This research did not receive any specific grant from funding agencies in the public, commercial, or not-for-profit sectors.

\section{Declarations}

Conflict of interest The authors declare no competing financial interests.

Ethical approval This work was conducted ethically in accordance with the World Medical Association Declaration of Helsinki. The Institutional Review Boards at Boston Children's Hospital and Brigham and Women's Hospital (shared protocol P00025766) and Boston Medical Center (H-35877) approved this work and did not require informed consent.

\section{References}

1. Overman RE Jr, Criss CN, Gadepalli SK (2019) Necrotizing enterocolitis in term neonates: a different disease process? J Pediatr Surg 54:1143-1146. https://doi.org/10.1016/j.jpedsurg.2019.02. 046

2. Stapleton GE, Eble BK, Dickerson HA, Andropoulos DB, Chang AC (2007) Mesenteric oxygen desaturation in an infant with congenital heart disease and necrotizing enterocolitis. Tex Heart Inst J 34:442-444

3. Ostlie DJ, Spilde TL, St Peter SD, Sexton N, Miller KA, Sharp RJ et al (2003) Necrotizing enterocolitis in full-term infants. J Pediatr Surg 38:1039-1042. https://doi.org/10.1016/s0022-3468(03) 00187-8

4. McElhinney DB, Hedrick HL, Bush DM, Pereira GR, Stafford PW, Gaynor JW et al (2000) Necrotizing enterocolitis in neonates with congenital heart disease: risk factors and outcomes. Pediatrics 106:1080-1087. https://doi.org/10.1542/peds.106.5.1080

5. Becker KC, Hornik CP, Cotten CM, Clark RH, Hill KD, Smith $P B$ et al (2015) Necrotizing enterocolitis in infants with ductaldependent congenital heart disease. Am J Perinatol 32:633-638. https://doi.org/10.1055/s-0034-1390349

6. Spinner JA, Morris SA, Nandi D, Costarino AT, Marino BS, Rossano JW et al (2020) Necrotizing enterocolitis and associated mortality in neonates with congenital heart disease: a multiinstitutional study. Pediatr Crit Care Med 21:228-234. https://doi. org/10.1097/PCC.0000000000002133

7. Lau PE, Cruz SM, Ocampo EC, Nuthakki S, Style CC, Lee TC et al (2018) Necrotizing enterocolitis in patients with congenital heart disease: a single center experience. J Pediatr Surg 53:914 917. https://doi.org/10.1016/j.jpedsurg.2018.02.014

8. Siano E, Lauriti G, Ceccanti S, Zani A (2019) Cardiogenic necrotizing enterocolitis: a clinically distinct entity from classical necrotizing enterocolitis. Eur J Pediatr Surg 29:14-22. https:// doi.org/10.1055/s-0038-1668144

9. Bubberman JM, van Zoonen A, Bruggink JLM, van der Heide M, Berger RMF, Bos AF et al (2019) Necrotizing enterocolitis associated with congenital heart disease: a different entity? J Pediatr Surg 54:1755-60. https://doi.org/10.1016/j.jpedsurg.2018.11.012

10. Kim WY, Kim WS, Kim IO, Kwon TH, Chang W, Lee E (2005) Sonographic evaluation of neonates with early-stage necrotizing enterocolitis. Pediatr Radiol 35:1056-1061. https://doi.org/10. 1007/s00247-005-1533-4

11. Miller SF, Seibert JJ, Kinder DL, Wilson AR (1993) Use of ultrasound in the detection of occult bowel perforation in neonates. $\mathrm{J}$ Ultrasound Med 12:531-535. https://doi.org/10.7863/jum.1993. 12.9 .531
12. Faingold R, Daneman A, Tomlinson G, Babyn PS, Manson DE, Mohanta A et al (2005) Necrotizing enterocolitis: assessment of bowel viability with color doppler US. Radiology 235:587-594. https://doi.org/10.1148/radiol.2352031718

13. Chen S, Hu Y, Liu Q, Li X, Wang H, Wang K et al (2019) Application of abdominal sonography in diagnosis of infants with necrotizing enterocolitis. Medicine 98:e16202. https://doi. org/10.1097/MD.0000000000016202

14. Cuna AC, Reddy N, Robinson AL, Chan SS (2018) Bowel ultrasound for predicting surgical management of necrotizing enterocolitis: a systematic review and meta-analysis. Pediatr Radiol 48:658-666. https://doi.org/10.1007/s00247-017-4056-x

15. McBride WJ, Roy S, Brudnicki A, Stringel G (2010) Correlation of complex ascites with intestinal gangrene and perforation in neonates with necrotizing enterocolitis. J Pediatr Surg 45:887-889. https://doi.org/10.1016/j.jpedsurg.2010.02.011

16. Epelman M, Daneman A, Navarro OM, Morag I, Moore AM, Kim JH et al (2007) Necrotizing enterocolitis: review of stateof-the art imaging findings with pathologic correlation. Radiographics 27:285-305. https://doi.org/10.1148/rg.272055098

17. Muchantef K, Epelman M, Darge K, Kirpalani H, Laje P, Anupindi SA (2013) Sonographic and radiographic imaging features of the neonate with necrotizing enterocolitis: correlating findings with outcomes. Pediatr Radiol 43:1444-1452. https://doi.org/10.1007/s00247-013-2725-y

18. Tracy SA, Lazow SP, Castro-Aragon IM, Fujii AM, Estroff JA, Parad RB et al (2020) Is abdominal sonography a useful adjunct to abdominal radiography in evaluating neonates with suspected necrotizing enterocolitis? J Am Coll Surg 230:903-911. https:// doi.org/10.1016/j.jamcollsurg.2020.01.027

19. Bohnhorst B, Kuebler JF, Rau G, Gluer S, Ure B, Doerdelmann M (2011) Portal venous gas detected by ultrasound differentiates surgical NEC from other acquired neonatal intestinal diseases. Eur J Pediatr Surg 21:12-17. https://doi.org/10.1055/s-00301265204

20. Harris PA, Taylor R, Minor BL, Elliott V, Fernandez M, O’Neal L et al (2019) The REDCap consortium: building an international community of software platform partners. J Biomed Inform 95:103208. https://doi.org/10.1016/j.jbi.2019.103208

21. Harris PA, Taylor R, Thielke R, Payne J, Gonzalez N, Conde JG (2009) Research electronic data capture (REDCap)-a metadatadriven methodology and workflow process for providing translational research informatics support. J Biomed Inform 42:377-381. https://doi.org/10.1016/j.jbi.2008.08.010

22. Alexander KM, Chan SS, Opfer E, Cuna A, Fraser JD, Sharif $S$ et al (2021) Implementation of bowel ultrasound practice for the diagnosis and management of necrotising enterocolitis. Arch Dis Child Fetal Neonatal Ed 106:96-103. https://doi.org/10.1136/ archdischild-2019-318382

23. Velazco CS, Fullerton BS, Hong CR, Morrow KA, Edwards EM, Soll RF et al (2017) Morbidity and mortality among "big" babies who develop necrotizing enterocolitis: a prospective multicenter cohort analysis. J Pediatr Surg 53:108-112. https://doi.org/10. 1016/j.jpedsurg.2017.10.028

24. Hamrick SEG, Sallmon H, Rose AT, Porras D, Shelton EL, Reese $\mathrm{J}$ et al (2020) Patent ductus arteriosus of the preterm infant. Pediatrics 146:e20201209. https://doi.org/10.1542/peds.2020-1209

25. Härkin P, Marttila R, Pokka T, Saarela T, Hallman M (2018) Morbidities associated with patent ductus arteriosus in preterm infants. Nationwide cohort study. J Matern Fetal Neonatal Med 31:2576-2583. https://doi.org/10.1080/14767058.2017.1347921

26. Fujii AM, Brown E, Mirochnick M (2002) Neonatal necrotizing enterocolitis with intestinal perforation in extremely premature infants receiving early indomethacin treatment for patent ductus arteriosus. J Perinatol 22:535-540. https://doi.org/10.1038/sj.jp. 7210795 
27. Grosfeld JL, Chaet M, Molinari F, Engle W, Engum SA, West $\mathrm{KW}$ et al (1996) Increased risk of necrotizing enterocolitis in premature infants with patent ductus arteriosus treated with indomethacin. Ann Surg 224:350-355. https://doi.org/10.1097/00000 658-199609000-00011
Publisher's Note Springer Nature remains neutral with regard to jurisdictional claims in published maps and institutional affiliations. 